\title{
STOKES PHENOMENON AND MATCHED ASYMPTOTIC EXPANSIONS*
}

\author{
A. B. OLDE DAALHUIS $\dagger$, S. J. CHAPMAN $\dagger$, J. R. KING $\ddagger$, J. R. OCKENDON†,
} AND R. H. TEW

\begin{abstract}
This paper describes the use of matched asymptotic expansions to illuminate the description of functions exhibiting Stokes phenomenon. In particular the approach highlights the way in which the local structure and the possibility of finding Stokes multipliers explicitly depend on the behaviour of the coefficients of the relevant asymptotic expansions.
\end{abstract} tion

Key words. Stokes' phenomenon, matched asymptotic expansions, Airy function, error func-

AMS subject classifications. $41 \mathrm{~A} 60,33 \mathrm{C} 10,33 \mathrm{~B} 20$

1. Introduction. The role of Stokes phenomenon in describing the asymptotic behaviour of an analytic function as its argument tends to an isolated singularity has been studied intensively in recent years (Berry [1], Berry and Howls [3], McLeod [4], Meyer [5], Olde Daalhuis and Olver [8], Olver [10], Paris [12], Paris and Wood [13]). As originally discussed by Stokes, the basic picture is that an asymptotic expansion of the function that is uniform in $\mathrm{ph} z$ can be constructed only if an exponentially small correction (in terms of distance from the singularity) is made as certain directions are traversed in the Argand diagram. These directions are called Stokes lines, and when the function under consideration is a complementary function of a certain class of linear holomorphic second-order differential equations, they are characterised as lines where one complementary function is maximally dominant over another. The Stokes lines are separated by other directions, called anti-Stokes lines, which are practically important because, across them, a complementary function switches from being dominant to subdominant; however, there is no nonuniformity in their vicinity, and all the action takes place near the Stokes lines.

What has emerged recently is the detailed structure of the behaviour in the vicinity of Stokes lines, at least for a class of functions whose asymptotic expansions diverge in a certain way. Thus, instead of the traditional asymptotic representation in terms of divergent expansions in different sectors, with discontinuous coefficients that are related by the Stokes connection formulae, the smoothness inherent in the analyticity can be restored, and this can be done universally in terms of error functions [1].

The purpose of this paper is to interpret this state of affairs in terms of theory of matched expansions (MAEs) (van Dyke [14]), not just with the aim of simplifying the representation of the above-mentioned new developments but also to pave the way for these developments to be exploited in problems other than the linear ordinary differential equations (ODEs) that have been considered hitherto.

To fix ideas, we will begin by recalling Stokes phenomenon for some linear ODEs where there are explicit integral representations. These examples will then be used

* Received by the editors January 19, 1994; accepted for publication (in revised form) October $31,1994$.

†Mathematical Institute, University of Oxford, 24-29 St. Giles, Oxford OX1 3LB, United Kingdom.

$\ddagger$ Department of Theoretical Mechanics, University of Nottingham, Nottingham NG7 2RD, United Kingdom. 
to motivate the construction of the MAE framework with which we will be working. It will first enable us to study the relevance of the phenomenon for a class of linear homogeneous ODEs in order to explain how to decide when connection formulae can be calculated explicitly rather than by numerical computation. Then we use the MAE format to present new results concerning the asymptotic behaviour of inhomogeneous ODEs. Finally, in the conclusion, we will be able to make some conjectures about the applicability of our framework to other ODEs and partial differential equations (PDEs).

A crucial aspect of our approach is the reinterpretation of Stokes phenomenon in terms other than that of maximal dominance of complementary functions of ODEs. One interpretation that emerges naturally from Berry [1] is that of the rapidity with which the difference between the solution of an ODE and its optimally truncated asymptotic expansion varies as $\mathrm{ph} z$ varies. This is tied in with the description of Stokes phenomenon not as a change in the coefficient of a complementary function but rather as a change in the remainder of the asymptotic expansion of the dominant complementary function in the region where this remainder is comparable with the subdominant complementary function. This remainder or error will have relatively smooth variation away from a Stokes line, and the change traditionally ascribed to the Stokes multiplier is a consequence of the rapid variation in this error rather than its cause. However, yet another characterisation has been proposed by Wright [15] in terms of the equality of the phase of certain solutions of linear PDEs when interpreted as waves, and this is the one that will emerge most naturally as a result of our investigation.

2. Example: The complementary error function. The complementary error function is a well-known function defined by

$$
\operatorname{erfc}(z)=\frac{2}{\sqrt{\pi}} \int_{z}^{\infty} e^{-t^{2}} d t, \quad z \in \mathbb{C} .
$$

It is an entire function with the following asymptotic behaviour:

$$
\begin{array}{rlrl}
\operatorname{erfc}(z) & \sim \frac{e^{-z^{2}}}{z \sqrt{\pi}} \sum_{s=0}^{\infty}(-1)^{s} \frac{(2 s) !}{s !\left(4 z^{2}\right)^{s}}, & & |\operatorname{ph}(z)|<\frac{3}{4} \pi \\
\operatorname{erfc}(z) \sim 2+\frac{e^{-z^{2}}}{z \sqrt{\pi}} \sum_{s=0}^{\infty}(-1)^{s} \frac{(2 s) !}{s !\left(4 z^{2}\right)^{s}}, & & |\operatorname{ph}(-z)|<\frac{3}{4} \pi
\end{array}
$$

as $|z| \rightarrow \infty$. Notice that both asymptotic expansions are valid in the sector $\frac{1}{4} \pi<$ $\operatorname{ph} z<\frac{3}{4} \pi$. So the asymptotic behaviour of $\operatorname{erfc}(z)$ is the infinite expansion of $(2.2 \mathrm{a})$, plus a constant. In the sector $-\frac{1}{4} \pi<\operatorname{ph} z<\frac{1}{4} \pi$, where $\exp \left(-z^{2}\right) / z \sqrt{\pi}$ is subdominant, this constant is 0 . In the sector $\frac{3}{4} \pi<\operatorname{ph} z<\frac{5}{4} \pi$, where, again, $\exp \left(-z^{2}\right) / z \sqrt{\pi}$ is subdominant, this constant is 2 . And in the sector $\frac{1}{4} \pi<\operatorname{ph} z<\frac{3}{4} \pi$, where $\exp \left(-z^{2}\right) / z \sqrt{\pi}$ is dominant, this constant changes from 0 to 2 (see Fig. 2.1).

We want to obtain the change of the asymptotic behaviour directly from the differential equation

$$
\frac{d^{2}}{d z^{2}} w(z)+2 z \frac{d}{d z} w(z)=0
$$

without use of (2.1). Both $\operatorname{erfc}(z)$ and the constant function are solutions of this differential equation. To obtain more information on the change in the sector $\frac{1}{4} \pi<$ 


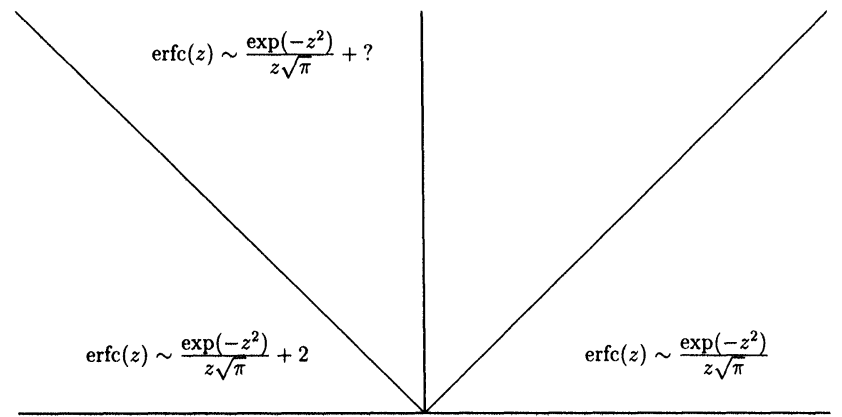

FIG. 2.1. The asymptotic behaviour of $\operatorname{erfc}(z)$ for $0 \leq \mathrm{ph} z \leq \pi$.

$\operatorname{ph} z<\frac{3}{4} \pi$, we truncate $(2.2 \mathrm{a})$ after $N$ terms:

$$
\operatorname{erfc}(z)=\frac{e^{-z^{2}}}{z \sqrt{\pi}} \sum_{s=0}^{N-1}(-1)^{s} \frac{(2 s) !}{s !\left(4 z^{2}\right)^{s}}+R_{N}(z) .
$$

The remainder $R_{N}(z)$ is a solution of

$$
\frac{d^{2}}{d z^{2}} R_{N}(z)+2 z \frac{d}{d z} R_{N}(z)=\frac{e^{-z^{2}}}{z \sqrt{\pi}} \frac{(-1)^{N}(2 N) !}{(N-1) ! 4^{N-1} z^{2 N}} .
$$

To show where the significant changes take place, we introduce polar coordinates (2.6)

$$
z=r e^{i \theta}, \quad 0<\theta<\pi, \quad \frac{d}{d z}=-\frac{i e^{-i \theta}}{r} \frac{d}{d \theta}, \quad \frac{d^{2}}{d z^{2}}=\frac{i e^{-2 i \theta}}{r^{2}} \frac{d}{d \theta}-\frac{e^{-2 i \theta}}{r^{2}} \frac{d^{2}}{d \theta^{2}},
$$

where we have deliberately written $\frac{d}{d z}$ in terms of the "fast" variable $\theta$ rather than the "slow" variable $r$. Equation (2.5) in terms of polar coordinates is

$$
\begin{aligned}
-\frac{e^{-2 i \theta}}{r^{2}} \frac{d^{2}}{d \theta^{2}} R_{N}(z)+ & \left(\frac{e^{-2 i \theta}}{r^{2}}-2\right) \frac{d}{d \theta} R_{N}(z) \\
& =\frac{\exp \left[-r^{2} e^{2 i \theta}+N \pi i-(2 N+1) i \theta\right](2 N) !}{\sqrt{\pi}(N-1) ! 4^{N-1} r^{2 N+1}} .
\end{aligned}
$$

The magnitude of the right-hand side of (2.7), as a function of $N$, is minimal for $N \sim r^{2}$. Therefore, we take $N=r^{2}+\alpha$, where $\alpha$ is bounded as $r \rightarrow \infty$. With this $N$ the right-hand side of (2.7) reads

$$
\begin{aligned}
& \frac{\exp \left[-r^{2}\left(e^{2 i \theta}+i(2 \theta-\pi)\right)+\alpha \pi i-(2 \alpha+1) \theta i\right]\left(2 r^{2}+2 \alpha\right) !}{\sqrt{\pi}\left(r^{2}+\alpha-1\right) ! 4^{r^{2}+\alpha-1} r^{2 r^{2}+2 \alpha+1}} \\
& \sim \frac{8 r}{\sqrt{2 \pi}} e^{-i \alpha(2 \theta-\pi)-i \theta} \exp \left[-r^{2}\left(e^{2 i \theta}+1+i(2 \theta-\pi)\right)\right]
\end{aligned}
$$

as $r \rightarrow \infty$. The dominant factor in the right-hand side of $(2.8)$ is $\mid \exp \left[-r^{2}\left(e^{2 i \theta}+1+\right.\right.$ $i(2 \theta-\pi))]$, and it is maximal at $\theta=\frac{1}{2} \pi$, where it is $\mathcal{O}(1)$, as $r \rightarrow \infty$. The value $\theta=\frac{1}{2} \pi$ has two other special properties. First, the phase of the second exponential is both zero and stationary at this point (so that the right-hand side ceases to be oscillatory as $r \rightarrow \infty$ in the vicinity of the Stokes line). Second, and most importantly 
for the present point of view, the right-hand side of (2.8) is independent of $\alpha$ when $\theta=\frac{1}{2} \pi$. This latter property allows us to use matched asymptotic expansions to solve for $R_{N}$ in the neighbourhood of the Stokes line. We write

$$
r=\frac{r_{0}}{\varepsilon}, \quad \theta=\frac{1}{2} \pi+\delta \theta_{0}
$$

where $\varepsilon$ and $\delta$ are the new small parameters. With the substitution of (2.9) into (2.7), we obtain

$$
\begin{aligned}
\frac{e^{-2 i \delta \theta_{0}}}{r_{0}^{2}} \frac{\varepsilon^{2}}{\delta^{2}} \frac{d^{2}}{d \theta_{0}^{2}} R_{N}(z)- & i\left(\frac{e^{-2 i \delta \theta_{0}}}{r_{0}^{2}} \frac{\varepsilon^{2}}{\delta}+\frac{2}{\delta}\right) \frac{d}{d \theta_{0}} R_{N}(z) \\
& \sim \frac{-8 r_{0} i}{\varepsilon \sqrt{2 \pi}} e^{-2 i \delta \theta_{0} \alpha-i \delta \theta_{0}} \exp \left[\frac{r_{0}^{2}}{\varepsilon^{2}}\left(e^{2 i \delta \theta_{0}}-1-2 i \delta \theta_{0}\right)\right] \\
& \sim \frac{-8 r_{0} i}{\varepsilon \sqrt{2 \pi}} \exp \left[-2 r_{0}^{2} \theta_{0}^{2} \frac{\delta^{2}}{\varepsilon^{2}}\right]
\end{aligned}
$$

as $\delta, \varepsilon \rightarrow 0$. From the final exponential we only obtain an interesting result when $\delta=\varepsilon$. Then the dominant terms are the $\varepsilon^{-1}$ terms, and we obtain

$$
\frac{d}{d \theta_{0}} R_{N}(z) \sim \frac{4 r_{0}}{\sqrt{2 \pi}} \exp \left(-2 r_{0}^{2} \theta_{0}^{2}\right)
$$

with the solution

$$
R_{N}(z) \sim A+\operatorname{erf}\left(\sqrt{2} r_{0} \theta_{0}\right), \quad z=\frac{r_{0}}{\varepsilon} e^{i\left(\frac{1}{2} \pi+\varepsilon \theta_{0}\right)}, \quad \varepsilon \rightarrow 0
$$

where $\operatorname{erf}(z)$ is the error function. Matching as $\theta_{0} \rightarrow-\infty$, the remainder $R_{N}(z)$ is exponentially small. Thence, $A=1$. Thus all the change in the constant term takes place in the neighbourhood of the Stokes line $\operatorname{ph} z=\frac{1}{2} \pi$, and the change reads

$$
R_{N}(z) \sim 1+\operatorname{erf}\left(\sqrt{2} r_{0} \theta_{0}\right)
$$

in agreement with [1]. Figure 2.2 shows the appearance of the constant term in the asymptotic behaviour of $\operatorname{erfc}(z)$ in the sector $0 \leq \operatorname{ph} z \leq 2 \pi$. Notice that the constant term appears at the Stokes line $\mathrm{ph} z=\frac{1}{2} \pi$, it is dominant in the sector $\frac{3}{4} \pi<\operatorname{ph} z<\frac{5}{4} \pi$, and it disappears at the Stokes line $\operatorname{ph} z=\frac{3}{2} \pi$.

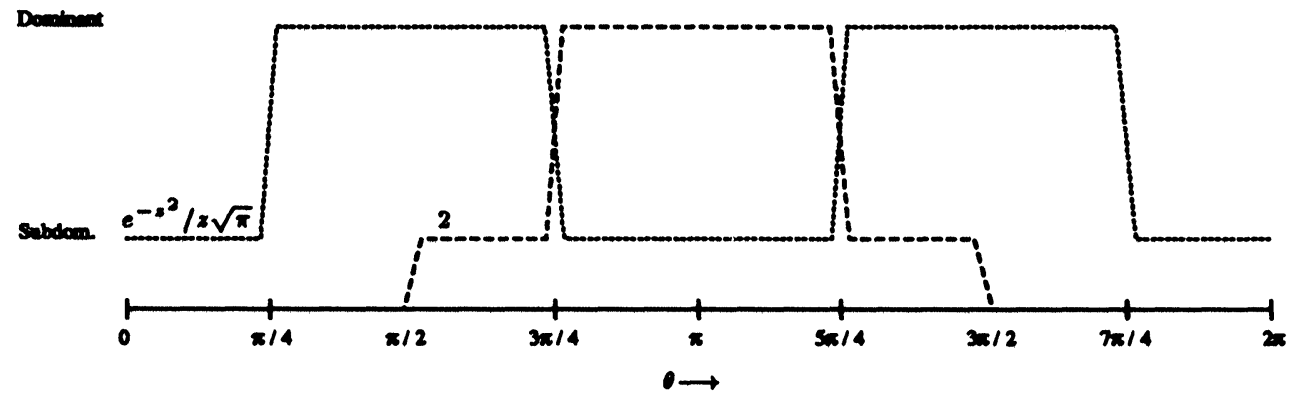

FIG. 2.2. The appearance of the constant term in the asymptotic behaviour of $\operatorname{erfc}(z)$. 
3. The Stokes phenomenon for solutions of a class of ODEs. The general homogeneous linear differential equation of the second order is given by

$$
\frac{d^{2}}{d z^{2}} v(z)+f(z) \frac{d}{d z} v(z)+g(z) v(z)=0
$$

We suppose that the point at infinity is an irregular singularity of rank 1 . The asymptotic theory of solutions of (3.1) in these circumstances is well known and will be found, for example, in Olver [9, Chap. 7, $\S \S 1-2]$. Without loss of generality we may assume that $f(z)$ and $g(z)$ can be expanded in the power series

$$
f(z)=1-\frac{\mu}{z}+\sum_{s=2}^{\infty} \frac{f_{s}}{z^{s}}, \quad g(z)=\sum_{s=2}^{\infty} \frac{g_{s}}{z^{s}}
$$

which converge for $|z| \geq \rho$. The two unique solutions of (3.1) have the following asymptotic behaviour:

$$
\begin{aligned}
& v_{1}(z) \sim e^{-z} z^{\mu} \sum_{s=0}^{\infty} \frac{a_{s}}{z^{s}}, \quad|\operatorname{ph}(z)| \leq \frac{3}{2} \pi-\nu \\
& v_{2}(z) \sim 1, \quad|\operatorname{ph}(z)-\pi| \leq \frac{3}{2} \pi-\nu .
\end{aligned}
$$

Here and elsewhere in the paper $\nu$ denotes an arbitrary small positive parameter. We choose $a_{0}=1$, and the other coefficients are determined by

$$
-s a_{s}=s(s-1-\mu) a_{s-1}+\sum_{m=0}^{s-1}\left(g_{s+1-m}-f_{s+1-m}\right) a_{m}-\sum_{m=0}^{s-2}(m-\mu) f_{s-m} a_{m}
$$

$s \geq 1$. A direct consequence of (3.2) is that $v_{1}\left(z e^{-2 \pi i}\right)$ is also a solution of (3.1), and note that $v_{1}(z)$ and $e^{2 \pi i \mu} v_{1}\left(z e^{-2 \pi i}\right)$ are dominant solutions in the sector $\frac{1}{2} \pi+\nu \leq$ $\operatorname{ph} z \leq \frac{3}{2} \pi-\nu$ and have exactly the same asymptotic expansion there. Thus there is a Stokes multiplier $C$ such that

$$
v_{1}(z)=e^{2 \pi i \mu} v_{1}\left(z e^{-2 \pi i}\right)+C v_{2}(z) .
$$

With this connection formula we obtain the following asymptotic behaviour for $v_{1}(z)$ :

$$
\begin{aligned}
& v_{1}(z) \sim e^{-z} z^{\mu}, \quad-\frac{1}{2} \pi<\operatorname{ph} z<\frac{1}{2} \pi, \quad \text { subdominant }, \\
& v_{1}(z) \sim e^{-z} z^{\mu}, \quad \frac{1}{2} \pi<\operatorname{ph} z<\frac{3}{2} \pi, \quad \text { dominant } \\
& v_{1}(z) \sim C, \quad \frac{3}{2} \pi<\operatorname{ph} z<\frac{5}{2} \pi, \quad \text { dominant. }
\end{aligned}
$$

Again, somewhere in the sector $\frac{1}{2} \pi<\operatorname{ph} z<\frac{3}{2} \pi$, new exponentially small terms appear. To obtain more information concerning the change in this sector, we truncate (3.3a) after $N$ terms

$$
v_{1}(z)=e^{-z} z^{\mu} \sum_{s=0}^{N-1} \frac{a_{s}}{z^{s}}+R_{N}(z)
$$


In the appendix we shall prove that the remainder is a solution of the following inhomogeneous differential equation:

$$
\begin{aligned}
\frac{d^{2}}{d z^{2}} R_{N}(z)+ & f(z) \frac{d}{d z} R_{N}(z)+g(z) R_{N}(z)=e^{-z} z^{\mu-N-1} N a_{N} \\
& -e^{-z} z^{\mu} \sum_{k=N+2}^{\infty} z^{-k} \sum_{m=0}^{N-1} a_{m}\left[g_{k-m}-f_{k-m}-(m-\mu) f_{k-1-m}\right] .
\end{aligned}
$$

Olver shows in [11] that

$$
a_{N} \sim \Lambda(-1)^{N} \Gamma(N-\mu) \quad \text { as } N \rightarrow \infty,
$$

where $\Lambda$ is a constant. From this result we obtain that the first term of the right-hand side of (3.8) is minimal for $N \sim|z|$. We take $N=|z|+\beta$, with $\beta$ bounded. In the appendix we shall show that with this $N$ we can estimate the right-hand side of (3.8) by its first term:

right-hand side of $(3.8) \sim e^{-z} z^{\mu-N-1} N a_{N}$

$$
\begin{aligned}
& \sim \Lambda \sqrt{\frac{2 \pi}{r}} e^{i \theta(\mu-1)-i \beta(\theta-\pi)} \exp \left(-r\left[e^{i \theta}+1+i(\theta-\pi)\right]\right), \\
& |z| \rightarrow \infty, \quad z=r e^{i \theta}, \quad N=r+\beta
\end{aligned}
$$

Note again that (3.10) is maximal, ceases to oscillate, and is independent of $\beta$ when $\theta=\pi$. Again, we use matched asymptotic expansions, but now with $r=r_{0} / \varepsilon$ and $\theta=\pi+\delta \theta_{0}$

$$
\begin{aligned}
-\frac{e^{-2 i \delta \theta_{0}}}{r_{0}^{2}} \frac{\varepsilon^{2}}{\delta^{2}} \frac{d^{2}}{d \theta_{0}^{2}} R_{N}(z) & +i\left((1+\mu) \frac{e^{-2 i \delta \theta_{0}}}{r_{0}^{2}} \frac{\varepsilon^{2}}{\delta}+\frac{e^{-i \delta \theta_{0}}}{r_{0}} \frac{\varepsilon}{\delta}+\cdots\right) \frac{d}{d \theta_{0}} R_{N}(z) \\
& +\left(\frac{g_{2} e^{-2 i \delta \theta_{0}}}{r_{0}^{2}} \varepsilon^{2}+\cdots\right) R_{N}(z) \\
& \sim \Lambda \sqrt{\frac{2 \pi \varepsilon}{r_{0}}} e^{i\left(\pi+\delta \theta_{0}\right)(\mu-1)-i \beta \delta \theta_{0}} \exp \left(\frac{r_{0}}{\varepsilon}\left(e^{i \delta \theta_{0}}-1-i \delta \theta_{0}\right)\right) \\
& \sim \Lambda \sqrt{\frac{2 \pi \varepsilon}{r_{0}}} e^{i \pi(\mu-1)} \exp \left(-\frac{1}{2} r_{0} \theta_{0}^{2} \frac{\delta^{2}}{\varepsilon}\right)
\end{aligned}
$$

as $\delta, \varepsilon \rightarrow 0$. From the final exponential we obtain that we have to take $\delta^{2}=\varepsilon$ in order to obtain an interesting balance. The dominant terms are the $\varepsilon^{\frac{1}{2}}$ terms, and we obtain

$$
i \frac{d}{d \theta_{0}} R_{N}(z) \sim \Lambda \sqrt{2 \pi} e^{i \pi(\mu-1)} r_{0}^{\frac{1}{2}} \exp \left(-\frac{1}{2} r_{0} \theta_{0}^{2}\right),
$$

with the solution

$$
R_{N}(z) \sim \Lambda 2 \pi i e^{i \pi \mu}\left[A+\frac{1}{2} \operatorname{erf}\left(\theta_{0} \sqrt{\frac{1}{2} r_{0}}\right)\right]
$$

as $\varepsilon \rightarrow 0$, with $z=\left(r_{0} / \varepsilon\right) \exp \left(i\left(\pi+\sqrt{\varepsilon} \theta_{0}\right)\right)$. Matching as $\theta_{0} \rightarrow-\infty$, we find that $R_{N}(z)$ is exponentially small. Thence, $A=\frac{1}{2}$. And from (3.6c) we obtain that $\Lambda 2 \pi i e^{i \pi \mu}=C$ such that the change in the neighbourhood of the Stokes line $\operatorname{ph} z=\pi$ is given by

$$
R_{N}(z) \sim C\left[\frac{1}{2}+\frac{1}{2} \operatorname{erf}\left(\theta_{0} \sqrt{\frac{1}{2} r_{0}}\right)\right]
$$

in agreement with [8]. 
Remark. We note that, had we not assumed the expansions (3.2) (which can be achieved by a transformation of the general equation (3.1)) but worked with (3.1) directly with general $f$ and $g$, then it would have been necessary to write $R_{N}=S v_{2}^{0}$, where $v_{2}^{0}$ is the leading-order behaviour of $v_{2}$, and formulate the equation for the Stokes multiplier $S$. In the case considered, $v_{2}^{0}=1$ and $R_{N}=S$. Note also, however, that the Stokes lines can be determined from the condition that the right-hand side of (3.10) is independent of $\beta$, for which it is not necessary to know the leading-order behaviour of the solution $v_{2}$ that is to be "switched on." Indeed, the leading-order behaviour of this second function can then be deduced; i.e., given only the asymptotic expansion of $v_{1}$, both the Stokes lines and leading-order behaviour of $v_{2}$ can be deduced without reference to the differential equation! In fact, for second-order equations that have solutions

$$
\begin{aligned}
& v_{1} \sim z^{\mu_{1}} e^{\gamma_{1} z^{k}} \sum_{n=0}^{\infty} \frac{c_{n} e^{i \eta n}}{z^{k n}} \\
& v_{2} \sim z^{\mu_{2}} e^{\gamma_{2} z^{k}}
\end{aligned}
$$

where $c_{n}$ is real and positive, there are Stokes lines switching on $v_{2}$ wherever $\frac{e^{i \eta}}{z^{k}}$ is real, and $\gamma_{1}-\gamma_{2}=\lim _{n \rightarrow \infty} \frac{n c_{n-1}}{c_{n}} e^{-i \eta}$. Of course, when we are dealing with secondorder equations, it is much easier in general to determine the $\gamma_{i}$ than the $c_{n}$. However, for higher-order equations, and in particular for PDEs, the asymptotic behaviour of the solution to be switched on may not be known beforehand.

4. The connection coefficient $C$. The result (3.9) describes the growth of the coefficients of asymptotic expansion (3.3a), where $\Lambda$ is a constant. At the end of the previous section we showed that $\Lambda=C e^{-i \pi \mu} / 2 \pi i$, where $C$ is the constant in connection formula (3.5). Thence

$$
a_{N} \sim C \frac{(-1)^{N}}{2 \pi i} e^{-i \pi \mu} \Gamma(N-\mu) \quad \text { as } N \rightarrow \infty .
$$

Since we have the recurrence relation (3.4), we can use (4.1) for the numerical computation of $C$, but in general it is very difficult to obtain the value of $C$ analytically. Indeed, only when (3.4) is simple enough for us to find $a_{N}$ analytically can (4.1) be used to obtain the exact value of $C$. For example, for the confluent hypergeometric functions, (3.4) is a two-term recurrence relation, but for these functions there are also different methods (e.g., by integral representations) that can be used to obtain the connection constants. There are, however, more complicated differential equations for which the result (4.1) can be used to obtain the exact value of $C$. Consider the equation

$$
\frac{d^{2}}{d z^{2}} v(z)+\left(1+\frac{1}{z}+\frac{\lambda}{z^{2}}\right) \frac{d}{d z} v(z)=0
$$

with the solutions

$$
\begin{aligned}
& v_{1}(z) \sim e^{-z} z^{-1} \sum_{s=0}^{\infty} \frac{a_{s}}{z^{s}}, \quad|\operatorname{ph} z| \leq \frac{3}{2} \pi-\nu, \\
& v_{2}(z)=1 .
\end{aligned}
$$

The recurrence relation for the coefficients can be written in the form $a_{0}=1$, and for $s \geq 1$

$$
s\left(a_{s}+s a_{s-1}\right)=\lambda\left(a_{s-1}+(s-1) a_{s-2}\right),
$$


from which we obtain

$$
a_{s}=(-1)^{s} s ! \sum_{k=0}^{s} \frac{(-\lambda)^{k}}{(k !)^{2}} \text {. }
$$

Hence, the constant $C$ in

$$
v_{1}(z)=v_{1}\left(z e^{-2 \pi i}\right)+C
$$

is

$$
C=\lim _{s \rightarrow \infty} \frac{-a_{s}}{(-1)^{s} s !}=-\sum_{k=0}^{\infty} \frac{(-\lambda)^{k}}{(k !)^{2}}=-J_{0}(2 \sqrt{\lambda}) .
$$

There are also cases where the connection constants can be written down almost by inspection. An example is the differential equation for the Airy function $\operatorname{Ai}(z)$, namely,

$$
\frac{d^{2}}{d z^{2}} u(z)-z u(z)=0
$$

This differential equation is not of the form (3.1), but the transformation $z \mapsto x^{\frac{2}{3}}$ leads to a differential equation of that form. From $\S 3$ we obtain that equation (4.7) has Stokes lines at $\operatorname{ph} z=0, \frac{2}{3} \pi$, and $\frac{4}{3} \pi$. The Airy function $\operatorname{Ai}(z)$ is the subdominant solution at $\operatorname{ph} z=0$. A second solution of (4.7) is $\operatorname{Ai}\left(z e^{-\frac{2}{3} \pi i}\right)$, which is the subdominant solution at $\operatorname{ph} z=\frac{2}{3} \pi$. These two solutions have the following asymptotic behaviour:

$$
\begin{aligned}
& \operatorname{Ai}(z) \sim \frac{1}{2 \sqrt{\pi}} e^{-\frac{2}{3} z^{\frac{3}{2}}} z^{-\frac{1}{4}}, \quad|\operatorname{ph}(z)| \leq \pi-\nu, \\
& \operatorname{Ai}\left(z e^{-\frac{2}{3} \pi i}\right) \sim \frac{e^{\frac{1}{6} \pi i}}{2 \sqrt{\pi}} e^{\frac{2}{3} z^{\frac{3}{2}}} z^{-\frac{1}{4}}, \quad\left|\operatorname{ph}(z)-\frac{2}{3} \pi\right| \leq \pi-\nu, \\
& \text { as }|z| \rightarrow \infty \text {. }
\end{aligned}
$$

It is now possible to obtain the connection formula for $\operatorname{Ai}(z)$ just from (3.5) and the fact that $\operatorname{Ai}(z)$ is an entire function. From (3.5) we obtain that

$$
\operatorname{Ai}(z)=e^{-\frac{1}{3} \pi i} \operatorname{Ai}\left(z e^{-\frac{4}{3} \pi i}\right)+C_{1} \operatorname{Ai}\left(z e^{-\frac{2}{3} \pi i}\right) .
$$

A similar argument gives

$$
\operatorname{Ai}(z)=C_{2} \operatorname{Ai}\left(z e^{\frac{2}{3} \pi i}\right)+e^{\frac{1}{3} \pi i} \operatorname{Ai}\left(z e^{\frac{4}{3} \pi i}\right) .
$$

Since the Airy function is an entire function, we know that $\operatorname{Ai}\left(z e^{-\frac{4}{3} \pi i}\right)=\operatorname{Ai}\left(z e^{\frac{2}{3} \pi i}\right)$ and that $\operatorname{Ai}\left(z e^{-\frac{2}{3} \pi i}\right)=\operatorname{Ai}\left(z e^{\frac{4}{3} \pi i}\right)$. Therefore, we obtain from (4.9) and (4.10) the connection formula

$$
\operatorname{Ai}(z)=e^{-\frac{1}{3} \pi i} \operatorname{Ai}\left(z e^{\frac{2}{3} \pi i}\right)+e^{\frac{1}{3} \pi i} \operatorname{Ai}\left(z e^{-\frac{2}{3} \pi i}\right) .
$$

Figure 4.1 shows the appearance of exponentially small terms in the asymptotic behaviour of $\operatorname{Ai}(z)$ in the sector $0 \leq \operatorname{ph} z \leq 2 \pi$.

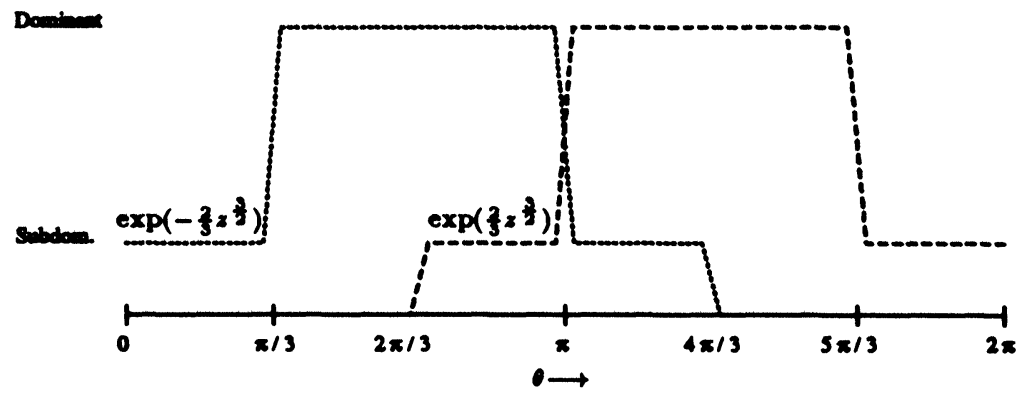

FIG. 4.1. The appearance of exponentially small terms in the asymptotic behaviour of $\operatorname{Ai}(z)$. 
Remark. In [9] the result (4.11) is obtained by the knowledge that there are constants $a, b$, and $c$, not all zero, such that

$$
a \operatorname{Ai}(z)+b \operatorname{Ai}\left(z e^{\frac{2}{3} \pi i}\right)+c \operatorname{Ai}\left(z e^{-\frac{2}{3} \pi i}\right)=0 .
$$

The values of $a, b$, and $c$ are obtained by looking at the asymptotic behaviour of the left-hand side of (4.12) at the Stokes lines at $\mathrm{ph} z=0, \pm \frac{3}{2} \pi$. Alternatively, we can use arguments involving the deformation of contour integral representations of $\operatorname{Ai}(z)$ to derive (4.11).

5. An inhomogeneous differential equation. In this section we show that our matched asymptotics method also works for inhomogeneous differential equations. We take as an example the differential equation

$$
\frac{d^{2}}{d z^{2}} w(z)+2 z \frac{d}{d z} w(z)=z \exp (-z)
$$

This is a rather special differential equation because the corresponding homogeneous differential equation (2.3) has an irregular singularity of rank 2 at infinity, which means that the solutions growth like $\exp \left(p_{2}(z)\right)$ as $z \rightarrow \infty$, where $p_{2}(z)$ is a polynomial of degree 2. Hence, there is a difference in the exponential growth of the solutions of the corresponding homogeneous differential equation and the right-hand side of (5.1). (Compare [9, Chap. 7, § 14].)

The general solution of $(5.1)$ is of the form

$$
w(z)=\operatorname{Aerfc}(z)+B+w_{p}(z)
$$

where $A$ and $B$ are arbitrary constants and $w_{p}(z)$ is a particular solution of (5.1). By variation of parameters we find the two particular solutions

$$
\begin{aligned}
w_{p_{ \pm}}(z) & =\frac{\sqrt{\pi}}{2} \operatorname{erfc}(z) \int_{z}^{ \pm i \infty} e^{t^{2}-t} t d t-\frac{\sqrt{\pi}}{2} \int_{z}^{\infty} e^{t^{2}-t} \operatorname{erfc}(t) t d t \\
& =-\frac{1}{2} e^{-z} \pm \frac{\pi i}{8} e^{-\frac{1}{4}} \operatorname{erfc}(z) \operatorname{erfc}\left(\mp i\left(z-\frac{1}{2}\right)\right)-\frac{\sqrt{\pi}}{4} \int_{z}^{\infty} e^{t^{2}-t} \operatorname{erfc}(t) d t
\end{aligned}
$$

From these integral representations we see that

$$
\begin{aligned}
& w_{p_{+}}(z) \sim-\frac{1}{2} e^{-z} \text { as } z \rightarrow \infty \text { in }-\frac{1}{4} \pi<\operatorname{ph} z<\frac{5}{4} \pi \\
& w_{p_{-}}(z) \sim-\frac{1}{2} e^{-z} \text { as } z \rightarrow \infty \text { in }-\frac{5}{4} \pi<\operatorname{ph} z<\frac{1}{4} \pi .
\end{aligned}
$$

When we substitute the formal solution $e^{-z} \sum_{s=0}^{\infty} a_{s} z^{-s}$ into (5.1) we obtain

$a_{0}=-\frac{1}{2}, \quad a_{1}=-\frac{1}{4}, \quad 2 a_{s+1}=(1-2 s) a_{s}+2(s-1) a_{s-1}+(s-2)(s-1) a_{s-2}, \quad s \geq 1$.

It is not difficult to show that ${ }^{1}$

$$
\begin{aligned}
& w_{p_{+}}(z) \sim e^{-z} \sum_{s=0}^{\infty} \frac{a_{s}}{z^{s}}, \quad-\frac{1}{4} \pi<\operatorname{ph} z<\frac{5}{4} \pi, \\
& w_{p_{-}}(z) \sim e^{-z} \sum_{s=0}^{\infty} \frac{a_{s}}{z^{s}}, \quad-\frac{5}{4} \pi<\operatorname{ph} z<\frac{1}{4} \pi,
\end{aligned}
$$

\footnotetext{
${ }^{1}$ E.g., by using the method that is used in [9, Chap. $\left.7, \S 14\right]$.
} 
as $z \rightarrow \infty$ and that ${ }^{2}$

$$
a_{s} \sim K(-1)^{s} \Gamma(s)
$$

as $s \rightarrow \infty$, where $K$ is a constant.

A direct consequence of $(5.3 \mathrm{~b})$ is the connection formula

$$
w_{p_{+}}(z)=w_{p_{-}}(z)+\frac{\pi i}{4} e^{-\frac{1}{4}} \operatorname{erfc}(z)
$$

With this connection formula we obtain the following asymptotic behaviour for $w_{p_{+}}(z)$ :

$$
\begin{aligned}
& w_{p_{+}}(z) \sim-\frac{1}{2} e^{-z}, \quad-\frac{1}{4} \pi<\operatorname{ph} z<\frac{3}{4} \pi, \quad \text { subdominant } \\
& w_{p_{+}}(z) \sim-\frac{1}{2} e^{-z}, \quad \frac{3}{4} \pi<\operatorname{ph} z<\frac{5}{4} \pi, \quad \text { dominant } \\
& w_{p_{+}}(z) \sim \frac{i \sqrt{\pi}}{4} e^{-\frac{1}{4}} \frac{e^{-z^{2}}}{z}, \quad \frac{5}{4} \pi<\operatorname{ph} z<\frac{7}{4} \pi, \quad \text { dominant. }{ }^{3}
\end{aligned}
$$

Hence, somewhere in the sector $\frac{3}{4} \pi<\operatorname{ph} z<\frac{5}{4} \pi$, new exponentially small terms appear. Again, we write

$$
w_{p_{+}}(z)=e^{-z} \sum_{s=0}^{N-1} \frac{a_{s}}{z^{s}}+R_{N}(z),
$$

and, with the method of $\S 4$, we obtain

$$
\frac{d}{d \theta_{0}} R_{N}(z) \sim i K \sqrt{2 \pi r_{0}} \exp \left(-\frac{1}{2} r_{0} \theta_{0}^{2}\right)
$$

where $z=\left(r_{0} / \varepsilon\right) \exp \left(i\left(\pi+\sqrt{\varepsilon} \theta_{0}\right)\right)$ and $N=\left(r_{0} / \varepsilon\right)+\beta, \beta$ bounded. Hence

$$
R_{N}(z) \sim K \pi i\left[A+\operatorname{erf}\left(\theta_{0} \sqrt{\frac{1}{2} r_{0}}\right)\right]
$$

as $\varepsilon \rightarrow 0$. Matching as $\theta_{0} \rightarrow-\infty, R_{N}(z)$ is exponentially small, and so $A=1$. When $\theta_{0} \rightarrow+\infty$, we obtain that $2 K \pi i \sim \frac{1}{4} \pi i e^{-\frac{1}{4}} \operatorname{erfc}(z) \sim \frac{1}{2} \pi i e^{-\frac{1}{4}}$ and thus $K=\frac{1}{4} e^{-\frac{1}{4}}$. Therefore, the change in the neighbourhood of the Stokes line $\operatorname{ph} z=\pi$ is given by

$$
R_{N}(z) \sim \frac{\pi i}{4} e^{-\frac{1}{4}}\left[1+\operatorname{erf}\left(\theta_{0} \sqrt{\frac{1}{2} r_{0}}\right)\right] .
$$

We now encounter a major obstacle that confronts efforts to generalise the results of $\S 3$. We were able to use our MAE method in the sector $\frac{3}{4} \pi<\operatorname{ph} z<\frac{5}{4} \pi$ for the very reason that the minimal remainder $R_{N}(z)$ is of the same order as $\operatorname{erfc}(z)$ as $z \rightarrow-\infty$. However, since in the sector $-\frac{1}{4} \pi<\operatorname{ph} z<\frac{1}{4} \pi$ the minimal remainder $R_{N}(z)$ is exponentially large compared with $\operatorname{erfc}(z)$, we cannot use our MAE method to determine

\footnotetext{
${ }^{2}$ E.g., by using the method that is used in [11].

${ }^{3}$ In the derivation of $(5.9 \mathrm{c})$ we use that all solutions of (5.1) are entire functions such that $(5.6 \mathrm{~b})$ is also valid for $\frac{5}{4} \pi<\operatorname{ph} z<\frac{7}{4} \pi$.
} 
where and how the function $\operatorname{erfc}(z)$ is switched on in this section. Hence, it is still an open problem what the Stokes smoothing in the sector $-\frac{1}{4} \pi<\operatorname{ph} z<\frac{1}{4} \pi$ should be. In this connection, we note that inhomogeneous second-order equations are intimately linked with homogeneous third-order equations. Indeed, differentiating (5.1) and eliminating the inhomogeneous terms yields a holomorphic third-order differential equation for $w$.

The failure to detect the other Stokes lines in the example above is due to the information about them being "swamped" by the information about the Stokes line that we were able to find. Such a situation will occur generically whenever two divergent asymptotic series are multiplied together. Consider, for example, the function $v(z)=E_{1}(z) E_{1}(-z)$, where $E_{1}$ is the exponential integral. Since $E_{1}$ has a Stokes line at $\operatorname{ph} z=\pi, v(z)$ will have Stokes lines at $\operatorname{ph} z=0$ and $\operatorname{ph} z=\pi$. Asymptotically expanding $v(z)$, we have

$$
v(z) \sim \frac{-1}{z^{2}}\left(\sum_{j=0}^{\infty} \frac{j !}{z^{j}}\right)\left(\sum_{k=0}^{\infty} \frac{(-1)^{k} k !}{z^{k}}\right) \sim \frac{-1}{z^{2}} \sum_{n=0}^{\infty} \frac{c_{n}}{z^{n}},
$$

where $c_{2 n}=(2 n+1) ! /(n+1), c_{2 n+1}=0$. Hence, in this case, the leading-order behaviour of the $c_{n}$ 's contains the information for both Stokes lines. However, if we consider instead the function

$$
\left(\sum_{j=1}^{\infty} \frac{j !}{z^{j}}\right)\left(\sum_{k=0}^{\infty} \frac{(-1)^{k} k !}{z^{k}}\right) \sim \sum_{n=0}^{\infty} \frac{c_{n}}{z^{n}}
$$

then $c_{n}=(n+1) ! /(n+2)-(-)^{n} n ! /(n+2)$, and the leading-order behaviour of $c_{n}$ contains no indication of the Stokes line at $\operatorname{ph} z=\pi$.

Things are even worse when the value of $n$ corresponding to optimal truncation differs for the two series. Consider, for example, the function $v(z)=E_{1}(z) \operatorname{erfc}(z)$. Then

$$
v(z) \sim \frac{e^{-z^{2}-z}}{z^{2} \sqrt{\pi}}\left(\sum_{j=0}^{\infty} \frac{j !}{z^{j}}\right)\left(\sum_{k=0}^{\infty} \frac{(-1)^{k}(2 k) !}{k ! 4^{k} z^{2 k}}\right) \sim \frac{e^{-z^{2}-z}}{z^{2} \sqrt{\pi}} \sum_{n=0}^{\infty} \frac{c_{n}}{z^{n}} .
$$

Again, $c_{n} \sim n$ ! as $n \rightarrow \infty$. However, whereas in the example above the second Stokes line could have been detected by considering the second term in the expansion of $c_{n}$ for large $n$, in this case the largest term corresponding to the tail of the asymptotic expansion of the error function to appear in the asymptotic expansion of $c_{n}$ is $\left((-1)^{\frac{n}{2}} n !\right) /\left(\frac{n}{2} ! 2^{n}\right)$, which is exponentially smaller than the leading-order behaviour of $c_{n}$.

We note that all of the examples given above are all solutions of homogeneous fourth-order holomorphic differential equations. Thus, for the solutions of inhomogeneous second-order equations and third- and higher-order homogeneous equations, one of the Stokes lines will be easy to detect from the leading-order behaviour as $n \rightarrow \infty$ of the coefficients in the asymptotic expansion of a solution, but the information about the other Stokes lines may be buried deep in the asymptotic expansions of these coefficients.

6. Discussion and conclusion. Using the format of matched asymptotic expansions, we have presented as unified an account as possible for the location of the Stokes lines of certain analytic functions and for the description of the asymptotic behaviour in the vicinity of these lines. When the functions satisfy certain ODEs, the 
local behaviour of $R_{N}(z)$, the error in the optimally truncated asymptotic expansion of the dominant solution, is affected by the dependence of $N$ on $z$ and the precise growth of $a_{n}$ as defined in (3.3a) for $n>N$. This behaviour is characterised by expressions such as (2.13) and (3.14), where both the argument of the error function and also the region over which $R_{N}(z)$ varies rapidly depend crucially on the properties of $N$ and $a_{n}$ : this region is wedge shaped in $\S 2$ but can, for example, be parabolic in the cases such as those considered in [10].

For the problems considered in $\S 3$, we have presented a systematic procedure for determing the requisite information about $R_{N}(z)$; in many cases this involves the numerical solution of the recurrence relation (3.4) (see [11]), and it seems likely that a necessary and sufficient condition for the existence of a closed formula for the Stokes multipliers is that a closed-form solution exists for (3.4). However, any attempt to apply our method to higher-order or inhomogeneous generalisations of those in $\S 3$ is bedeviled by the possibility of there being a hierarchy of Stokes lines. Hence it is appropriate to conclude with a summary of the different characterisations of Stokes lines that are available with the aim of highlighting those which are likely to be of most value for more general problems.

By focusing on the remainder $R_{N}(z)$, we have been able to assert that on a Stokes line

(i) (3.10) is maximal;

(ii) the phase of (3.10) and its $\theta$-derivative are independent of $r$;

(iii) (3.10) is independent of $\beta$.

Moreover, the discussion at the end of $\S 3$ has shown that (iii) above can sometimes allow us to identify Stokes lines directly from the asymptotic expansion of a function without reference to any differential equation. However, reverting to the examples in $\S 3$, we can consider what would have happened to (3.10) had we not made the assumption (3.2). Then, after transforming in accordance with the remark after (3.14), the right-hand side of (3.8) would have involved a factor $\exp \left(u_{1}-u_{2}-\left|u_{1}-u_{2}\right|\right)$, where the phases $u_{i}$ are such that $v_{i} \sim \exp \left(u_{i}\right)$ as $|z| \rightarrow \infty$. Hence, on the Stokes line

(iv) $\Re\left(u_{1}\right)-\Re\left(u_{2}\right)$ is maximal when considered as a function of $\theta$ on $r=$ constant;

(v) $\Im\left(u_{1}\right)=\Im\left(u_{2}\right)$.

This last characterisation coincides with the definition of Stokes phenomenon given by Wright [15] in his Fourier analysis of linear wave propagation (see also Berry and Howls [2]). Indeed, if it is possible to write a wave field in the frequency domain as a Fourier integral, then it is clear that a new wave is switched on whenever a steepest descent path crosses a singularity or a new stationary point, in which case (iv) and (v) are clearly satisfied. In this spirit we can, if we consider holomorphic functions of two complex variables, give a physical interpretation of Stokes phenomenon that is quite different from the consideration of remainders in asymptotic expansions and is in the spirit of Meyer [6].

Suppose a solution of Helmholtz's equation, say, with large wavenumber $k$ is approximated using ray theory in two dimensions. The associated rays will, in general, be both real and complex; while it is a simple matter to trace the phase and amplitude along real rays, the same is not true for complex rays, which are best thought of as twodimensional manifolds in the four-dimensional complexified space of the independent variables. On these complex manifolds, the amplitude can vary by exponentially large amounts in terms of $k$, a typical situation being near a caustic. On the bright side of a caustic there are two families of real rays, and on the dark side there is one family of complex rays carrying exponentially small amplitude. However, an exponentially large amplitude wave field could also exist on the dark side in principle, and some rule has to 
be prescribed for legislating against complex rays carrying such large amplitude. Now it is natural to characterise Stokes "surfaces" as those points of $\mathbb{C}^{2}$ where two rays can exist with equal "phases" (i.e., the respective waves are $\exp \left(i k u_{j}\right)$, where $\Re u_{1}=\Re u_{2}$ ). These points form a three-dimensional manifold across which exponentially small wave fields can be switched on or off as in $\S 2$. The details of the generalisation of that discussion to this higher dimensional situation are too complicated to give here, but it is clear that the information that a complex ray carries en route to real space is profoundly affected by what happens at the intersection of that ray with the Stokes surface. This is exactly analogous to the change of dominance that occurs on antiStokes lines being profoundly affected by what happened at a remote Stokes line. Hence a knowledge of the position of the Stokes surface and the switching rules that apply across it are vital if complex rays are to be of practical value. Incidentally, the connection formulae for any point of the Stokes surface emanating from a caustic can be read from an argument similar to that leading to (4.11); locally, near the caustic, the wavefield is, of course, described by an Airy function.

We conclude by mentioning that there has also been much recent research on the effect of nonlinearity on Stokes phenomenon (see, e.g., Novokshenov [7]). Although nonlinear connection formulae exist, say, for Painlevé II, they seem never to have been analysed by MAEs; indeed, it seems possible that nonlinearity may completely swamp the delicate switching of exponentially small terms that underlies Stokes phenomenon.

Appendix. We use the notations of $\S 3$, and we define

$$
\tilde{v}_{1}(z)=e^{-z} \sum_{s=0}^{N-1} \frac{a_{s}}{z^{s-\mu}}
$$

such that

$$
v_{1}(z)=\tilde{v}_{1}(z)+R_{N}(z)
$$

When we use (3.4) several times, we obtain

$$
\begin{aligned}
& \frac{d^{2}}{d z^{2}} \tilde{v}_{1}(z)+f(z) \frac{d}{d z} \tilde{v}_{1}(z)+g(z) \tilde{v}_{1}(z) \\
&=e^{-z} z^{\mu} {\left[\frac{N(N-1-\mu) a_{N-1}}{z^{N+1}}-\sum_{k=N+1}^{\infty} z^{-k} \sum_{m=0}^{N-1} a_{m}\left(f_{k-m}-g_{k-m}\right)\right.} \\
&\left.-z^{-(N+1)} \sum_{m=0}^{N-2}(m-\mu) a_{m} f_{N-m}-\sum_{k=N+2}^{\infty} z^{-k} \sum_{m=0}^{N-1}(m-\mu) a_{m} f_{k-1-m}\right] \\
&=_{*} e^{-z} z^{\mu}\left[-\frac{N a_{N}}{z^{N+1}}+\sum_{k=N+2}^{\infty} z^{-k} \sum_{m=0}^{N-1} a_{m}\left[g_{k-m}-f_{k-m}-(m-\mu) f_{k-1-m}\right]\right]
\end{aligned}
$$

where in $*$ we have used (3.4) with $s=N$. The combination of (A.2) with (A.3) leads to (3.8). To estimate the infinite sum in the final line of (A.3), we use

$$
\begin{aligned}
\left|a_{m}\right| & \leq K_{1}|\Gamma(m-\mu)|, & & m \geq 0 \\
\left|f_{j}\right|,\left|g_{j}\right| & \leq K_{2} \rho^{j}, & & j \geq 0
\end{aligned}
$$


where $K_{1}$ and $K_{2}$ are constants. To obtain (A.4a), we use (3.9), and to obtain (A.4b), we use the fact that the expansions of (3.2) converge for $|z| \geq \rho$. We substitute (A.4) into

(A.5)

$$
\begin{aligned}
\left|\sum_{k=N+2}^{\infty} z^{-k} \sum_{m=0}^{N-1} a_{m}\left[g_{k-m}-f_{k-m}-(m-\mu) f_{k-1-m}\right]\right| & \\
& \leq K_{1} K_{2} \sum_{k=N+2}^{\infty}\left(\frac{\rho}{|z|}\right)^{k} \sum_{m=0}^{N-1} \frac{|\Gamma(m-\mu)|}{\rho^{m}}\left[2+\frac{m+|\mu|}{\rho}\right] \\
& =K_{1} K_{2}\left(\frac{\rho}{|z|}\right)^{N+2} \frac{|z|}{|z|-\rho} \sum_{m=0}^{N-1} \frac{|\Gamma(m-\mu)|}{\rho^{m}}\left[2+\frac{m+|\mu|}{\rho}\right] \\
& \leq K_{1} K_{2}\left(\frac{2 \rho^{2}+(N-1+|\mu|) \rho}{|z|^{N+2}}\right) \frac{|z|}{|z|-\rho}|\Gamma(N-\mu)| \\
& \leq \sum_{*}^{N-1} \frac{K_{1} K_{2}\left(\frac{2 \rho^{2}+(N-1+|\mu|) \rho}{|(m-\mu)(m+1-\mu) \cdots(N-1-\mu)|}\right) \frac{|z|}{|z|-\rho}|\Gamma(N-\mu)| K(\mu, \rho) \frac{N \rho}{|N-1-\mu|}}{|z|^{N+2}} \\
& =\frac{N}{|z|^{N+2}}|\Gamma(N-\mu)| \mathcal{O}(1)
\end{aligned}
$$

as $|z| \rightarrow \infty$. In $*$ we have used

$$
\frac{\rho^{N-m}}{|(m-\mu)(m+1-\mu) \cdots(N-1-\mu)|} \leq K(\mu, \rho) \frac{\rho}{|N-1-\mu|},
$$

$m=0,1, \ldots, N-1$, where $K(\mu, \rho)$ does not depend on $N$. From (3.9) we obtain

$$
\left|\frac{N a_{N}}{z^{N+1}}\right| \sim \Lambda \frac{N}{|z|^{N+1}}|\Gamma(N-\mu)|
$$

as $N \sim|z| \rightarrow \infty$. The combination of (A.7) and (A.5) leads to the first line of (3.10).

\section{REFERENCES}

[1] M. V. Berry, Uniform asymptotic smoothing of Stokes's discontinuities, Proc. Roy. Soc. London Ser., A422 (1989), pp. 7-21.

[2] M. V. BERRY AND C. J. HowlS, Stokes surfaces of diffraction catastrophes with codimension three, Nonlinearity, 3 (1990), pp. 281-291.

[3] — - Hyperasymptotics for integrals with saddles, Proc. Roy. Soc. London Ser. A, A434 (1991), pp. 657-675.

[4] J. B. MCLEOD, Smoothing of Stokes discontinuities, Proc. Roy. Soc. London Ser. A, A437 (1992), pp. 343-354.

[5] R. E. MEYER, A simple explanation of the Stokes phenomenon, SIAM Rev., 31 (1989), pp. 435445.

[6] - On exponential asymptotics for nonseparable wave equations, I: Complex geometrical optics and connection, SIAM J. Appl. Math., 51 (1991), pp. 1585-1601. 
[7] V. Y. Novokshenov, Nonlinear stokes phenomenon for the second Painlevé equation, Phys. D, 63 (1993), pp. 1-7.

[8] A. B. Olde DAAlhuis AND F. W. J. Olver, Exponentially-improved asymptotic solutions of ordinary differential equations II: Irregular singularities of rank one, Proc. Roy. Soc. London Ser. A, A445 (1994), pp. 39-56.

[9] F. W. J. Olver, Asymptotics and Special Functions, Academic Press, London, New York, 1974.

[10] - On Stokes' phenomenon and converging factors 329-355, in Asymptotic and Computational Analysis, R. Wong, ed., Marcel Dekker, New York, 1990.

[11] - Asymptotic expansions of the coefficients in asymptotic series solutions of linear differential equations, Methods Appl. Analysis, 1 (1994), pp. 1-13.

[12] R. B. PARIS, Smoothing of the Stokes phenomenon for high-order differential equations, Proc. Roy. Soc. London Ser. A, A436 (1992), pp. 165-186.

[13] R. B. PARIS AND A. D. WoOD, Stokes phenomenon demystified, IMA Bulletin, 31 (1995), pp. $21-28$.

[14] M. D. VAN Dyke, Perturbation Methods in Fluid Mechanics, Parabolic Press, Stanford, 1975.

[15] F. J. Wright, The Stokes set of the cusp diffraction catastrophe, J. Phys. A, 13 (1980), pp. 2913-2928. 